Questions vives

\section{Questions Vives}

Recherches en éducation

$N^{\circ} 28 \mid 2017$

De l'indifférenciation à la différenciation

\title{
Distinguer compréhension et interprétation du texte : le sujet-lecteur, entre découverte de soi et apprentissage d'une posture
}

\section{Emmanuel Nal}

\section{(2) OpenEdition \\ Journals}

Édition électronique

URL : http://journals.openedition.org/questionsvives/2521

DOI : 10.4000/questionsvives.2521

ISSN : $1775-433 \mathrm{X}$

Éditeur

Université Aix-Marseille (AMU)

Édition imprimée

Date de publication : 29 décembre 2017

ISBN : 978-2-912643-52-0

ISSN : 1635-4079

\section{Référence électronique}

Emmanuel Nal, « Distinguer compréhension et interprétation du texte : le sujet-lecteur, entre découverte de soi et apprentissage d'une posture », Questions Vives [En ligne], № 28 | 2017, mis en ligne le 15 novembre 2018, consulté le 10 décembre 2020. URL : http://journals.openedition.org/ questionsvives/2521; DOI : https://doi.org/10.4000/questionsvives.2521

Ce document a été généré automatiquement le 10 décembre 2020.

\section{c)}

Questions Vives est mis à disposition selon les termes de la licence Creative Commons Attribution Pas d'Utilisation Commerciale - Pas de Modification 4.0 International. 


\title{
Distinguer compréhension et interprétation du texte : le sujet- lecteur, entre découverte de soi et apprentissage d'une posture
}

\author{
Emmanuel Nal
}

1 Réunir ce qui faisait l'objet d'une distinction, se méprendre en prenant une chose pour une autre, ressentir l'embarras d'une maladresse : expliciter les différentes acceptions du mot « confusion » s'apparente presque à un récit. C'est un peu l'expérience de l'élève qui découvre cette annotation en marge de sa composition et n'en maîtrise pas toujours le sens - « qu'ai-je donc confondu, et avec quoi ? » - même s'il perçoit, du moins, la présence d'une forme d'erreur. Mais il arrive que les élèves manifestent le souci de la prévenir. En cours de lettres de première supérieure, à la suite d'un exercice d'herméneutique littéraire particulièrement spectaculaire, notre professeur avait voulu démontrer que le style de Balzac était marqué par un solipsisme qui le faisait se mettre en scène lui-même dans ses romans. À cet effet, il avait convoqué plusieurs descriptions de paysages dans le Père Goriot ou dans Le Lys dans la vallée dont les courbes ne manquaient pas selon lui de tracer l'initiale «B» du nom de l'auteur. À la suite de son argumentaire, présenté avec une grande conviction, nous fûmes quelques élèves à poser « la » question : «Peut-on (et comment) être sûrs que c'est effectivement cela que l'auteur a voulu dire? " Ce souci de nombre de jeunes étudiants en littérature - qui manifeste aussi bien une inquiétude qu'une volonté de respecter le texte et son auteur - traduit comme un entre-deux: jusqu'où peut aller la liberté interprétative que nous donne un texte ? Où commencerait un «délire herméneutique » $(E c 0,2016)$ qui ne ferait dire au texte guère que ce qu'on veut lui faire dire et traduirait un skhizein, une rupture avec le texte comme altérité ? C'est peut-être parce qu'ils éprouvent - au double sens de ressentir et d'être mis à l'épreuve - un être du texte que des lecteurs en viennent à s'interroger ainsi. Le problème est au moins autant intellectuel que moral : qu'est-ce qu'un lecteur, et de quelle manière peut-il se situer face au texte? À partir de cette question première, notre réflexion s'organisera autour des deux concepts de compréhension et d'interprétation parfois sujets à 
confusion - d'autant qu'ils se trouvent mis en pratique et font l'objet d'évaluations en contexte scolaire - et dont nous estimons la distinction propice pour un lecteur amené à s'interroger sur sa posture face au texte. Le terme de posture, qui désigne au sens propre une manière de positionner ses membres, de "se tenir" pour pratiquer une activité, " met toujours l'accent sur une forme de singularité, un engagement du je » (Rouzel, 2016). Or, comment exprimer sa subjectivité grâce au texte sans la substituer à l'altérité du texte ? L'idée qui oriente notre démarche est que l'apprentissage de la lecture intègre aussi une problématique de la posture de lecteur, dans la mesure où ce dernier manifeste le souci de concilier un art de la présence (sa subjectivité met en lumière quelque chose du sens du texte) et de la juste distance (pour ne pas réduire le texte au seul reflet de sa subjectivité). Notre réflexion choisit de s'ancrer dans le contexte scolaire souvent déterminant dans l'expérience littéraire - en l'occurrence, à partir d'observations en lycée d'enseignement général auprès de deux classes de seconde et d'une classe de première $L$ dans le cadre de leurs cours de français. Ces observations menées sur une année scolaire, entre les mois de janvier et de mai, nous ont permis de mesurer la récurrence de certains habitus, en particulier une forme de culture de la restitution de ce que les élèves présumaient "attendu» par l'enseignant. Compréhensible, ce réflexe finissait cependant par les mettre particulièrement en difficulté lorsqu'ils devaient composer leur propre texte. L'idée d'une éducation au texte cherche ici comment favoriser une relation au texte écrit par un tiers comme au texte à écrire. Nous pensons que l'apprentissage d'une posture de lecteur est solidaire d'une forme de travail sur soi qui se mène tout au long de la vie; c'est la raison pour laquelle cette réflexion peut aussi convier d'autres lecteurs à une forme de retour réflexif sur leur expérience littéraire. Pour envisager un tel apprentissage, nous commencerons par problématiser l'expression « apprendre à lire » pour montrer comment elle amène à s'interroger sur ce que signifie comprendre un texte. Nous tenterons de montrer qu'une compréhension de l'altérité est complémentaire d'un «se comprendre soi-même » devant le texte, en nous fondant sur les travaux de Ricœur. Par le texte se noue une relation paradoxale: comment comprendre ce qui se joue dans une intersubjectivité différée et tâtonnante, actualisée par le texte comme tiers, entre le lecteur et l'auteur? Pour Umberto Eco, loin d'être un objet fini, le texte est bien au contraire un objet "ouvert " que le lecteur ne peut se contenter de recevoir passivement et qui implique, de sa part, un travail d'invention et d'interprétation. C'est à partir de ces travaux que nous essaierons d'éclairer les enjeux propres de l'interprétation et de proposer des éléments pour une approche pédagogique du travail interprétatif.

\section{Du texte au sujet-lecteur : qui « produit » qui ?}

\subsection{Un itinéraire des mots vers les idées}

2 L'apprentissage de la lecture tient-il véritablement ses promesses? Greisch semble en douter lorsqu'il écrit: "À peine avons-nous acquis la compétence de lire que nous la perdons déjà de vue ». Et d'ajouter : " "Quelle est la chose la plus difficile ?" se demande Goethe. Et il répond : "Celle qui paraît la plus facile. Voir de nos yeux ce qui est sous nos yeux" " (Greisch, 2006, p.58). La première activité de déchiffrage, souvent qualifiée d'« apprentissage de la lecture ", pour nécessaire qu'elle soit, n'épuise pas tous les mystères du texte. Dès sa Logique, Wolff décrivait certains caractères faisant du texte un 
clair-obscur : "On ne lit un livre que dans le dessein de savoir ce qu'il contient. Deux choses sont donc nécessaires pour cet effet. Il faut bien comprendre l'auteur et bien retenir les choses qu'il avance» (Wolff, 1736, p. 215). Cependant, comment attribuer de manière avisée les mots aux idées dont ils sont les supports? « Pour bien comprendre le sens d'un auteur ", écrit encore Wolff, « il faut avoir surtout soin de ne lier aux mots dont il se sert, que les idées qu'il y attache lui-même. Sans cela vous risqueriez de donner un faux sens à ses expressions, et de lui faire dire tout autre chose qu'il ne dit; ce qui n'arrive par malheur que trop souvent. [...] Mais comme un mot peut avoir diverses significations, il arrive aussi souvent que le même mot n'a pas toujours une seule et même signification dans le même livre, quoique l'auteur se l'imagine » (p. 216-217). Si l'on ose écrire, à la suite de ce que développe Wolff (1736), Babel existait bien avant Babel et la confusion avait déjà de quoi être présente entre pratiquants d'une même langue, puisque malgré la communauté d'un lexique, le mot s'environne pour chacun d'une valeur, de connotations, parfois d'un esthétisme faisant que l'on ignore à quel point notre propre compréhension est partagée par l'interlocuteur. Baudelaire peut certes écrire qu'« Il y a dans le mot, dans le verbe, quelque chose de sacré qui nous défend d'en faire un jeu de hasard. Manier savamment une langue, c'est pratiquer une espèce de sorcellerie évocatoire » (Baudelaire, 1993, p.117), qui rencontre néanmoins ses limites lorsque l'auteur est confronté aux indicibles qui n'offrent prise au lexique. La sorcellerie comme le charme opèrent d'ailleurs dans un clair-obscur qu'ils ne cherchent pas à résorber. Dans la mesure où le travail d'écriture "ne donne que des mots » et qu'« en les donnant avec l'origine et le mystère qui sont en eux [il] convie [...] à posséder ce que disent les mots » (Renard, 1970, p. 34), il comporte par essence le risque de malentendus avec le lecteur, lequel ignore à quel point il est initié à ce mystère et à quel point il " possède » les mots choisis par l'auteur; la marge que représente cet indiscernable peut déjà induire des confusions et c'est ce dont le lecteur s'inquiète lorsqu'il se demande s'il a " bien compris » un texte.

\subsection{Quelques enjeux d'une " compréhension »}

Que veut dire et que suppose une compréhension d'un texte? Vandendorpe relève préalablement que la compréhension s'appréhende en lien avec une quête de sens par le lecteur. De prime abord, le lecteur pourrait être tenté de penser que comprendre, c'est reconstituer un sens «qui préexisterait à la saisie qu'on peut en faire: on cherche "le sens d'un texte" [...]. Normalement implicite et tenu pour présent, toile de fond préexistant à toute activité discursive, il forme l'horizon sur lequel s'échafaudent nos jugements » (Vandendorpe, 1991, p. 96). Comprendre, ce n'est cependant pas reconstituer du sens pour Vandendorpe. Au contraire, c'est mener, dans l'exercice d'une lecture, «l'activité cognitive qui crée [le sens]» (Vandendorpe, 1998, p. 70). Il y aurait pour Vandendorpe une création originale dans la compréhension, dès la compréhension, qu'il ne faudrait pas confondre avec sa restitution a posteriori, la signification, laquelle «ne constitue que la paraphrase que l'on peut donner de sa compréhension » (p. 70). Or, à la question « Qu'avez-vous compris du texte? ?, les élèves-lecteurs se demandent souvent ce qu'il «faut» restituer du texte, ce qui serait attendu que l'on en dise, sans doute parce qu'ils redoutent particulièrement contresens et autres faux sens. Ils proposent ainsi des réponses de la forme «l'auteur veut dire que », ce qui nous parait engendrer deux grands problèmes. 
4 Le premier fait suite à un souci, louable, de respect du texte qui tend à faire rechercher la norme qui y serait cachée, alors même que le texte n'est pas conçu par son auteur comme le lieu de toutes les réponses. Ce faisant, on risque de s'abuser sur la prétention du texte comme sur l'intention d'un auteur qui n'entend pas nécessairement se livrer entièrement ni tout dire, comme Kierkegaard se plaît à l'écrire :

Après ma mort, personne (et c'est ma consolation) ne trouvera dans mes papiers une seule indication sur ce qui a proprement rempli ma vie; ne trouvera la note gravée au plus profond de moi-même, qui explique tout et fait souvent de ce que le monde appelait des bagatelles, des événements pour moi d'une immense importance, et une bagatelle à mes yeux, quand j'enlève la note secrète qui l'explique (Kierkegaard, 1963, p. 392).

5 Si l'on identifie l'intention créatrice de l'auteur qui préside à l'écriture du texte et se déploie au fur et à mesure des lignes comme la seule chose à découvrir de ce texte, on risque d'exclure par avance toute autre possibilité de cheminement personnel avec le texte et de se couper d'une réflexion quant à une posture de lecteur et le rôle spécifique qui pourrait être le sien. Ce serait là le second problème : croire qu'une compréhension rigoureuse du texte suppose de s'interdire d'exister devant lui.

Devenir lecteur, ce n'est donc pas seulement maîtriser une technique de décryptage des graphèmes - qui en est certes une condition nécessaire - cela suppose aussi d'être sensibilisé aux manières de travailler (avec) le texte, d'avoir perçu la différence entre comprendre et interpréter pour les avoir expérimentés, d'avoir défini et intériorisé une posture de lecteur, par laquelle il manifeste sa subjectivité au sein d'une relation triangulaire et complémentaire avec l'œuvre et l'auteur. Cela suppose enfin d'avoir dissipé les confusions liées à ce que lire et être lecteur veulent dire : en cela, nous pensons que cet apprentissage donne matière à une forme d'éducation au texte. Si l'on convient en effet qu'il y a dans l'éducation au sens global du terme une volonté de « donner à tous les mêmes moyens pour se comprendre en promouvant, dans ce mouvement même, la constitution de leurs différences" (Danvers, 1994, p. 97), une éducation au texte s'enracinerait dans un projet philosophique. Philosophique parce que favorisant une réflexion sur le rapport au sens initié par le texte; philosophique parce qu'analytique, de manière à éviter les confusions précédemment relevées et parce qu'elle se veut émancipatrice pour lecteur. Philosophique parce qu'elle porte l'enjeu éthique de l'équilibre d'une relation avec l'altérité du texte et l'autre de l'auteur.

7 La première tâche d'une telle éducation pourrait donc consister à expliciter en quoi consiste le comprendre. Dans une éducation philosophique au texte, nous pensons qu'une " compréhension»s'envisage à partir d'une prise de conscience propédeutique. Elle conduirait d'abord à renoncer par avance à toute «idéologie du texte absolu » (Greisch, 1987, p.6), s'il est vrai que «l'absolu littéraire n'existe pas» (Jacques, 2007, p. 76), autrement dit qu'en se donnant à lire, le texte ne donne pas que lui-même, et qu'il ne peut donc normer seul la lecture qui en est faite. Cette forme de relativité du texte s'entend de deux manières. D'une part, dans la mesure où le lexique n'existe pas hors de celui qui le mobilise; il est donc incarné et porte l'empreinte des sujets - ceux qui l'emploient, ceux qui le reçoivent. Un texte littéraire ou philosophique ne saurait donc se concevoir comme seul support de ce que l'auteur " a dit » car il excède déjà ce dire par son existence une fois mis en forme à travers tous les « éléments textuels qui excèdent le texte dans le texte lui-même» (Greisch, 1987, p.6) et qui rendent possible un «transcendantal du texte " (Jacques, 2007). D'autre part, dans la mesure où, comme le précise encore Jacques, «Un avertissement de l'auteur, un éditorial nous parlent du 
texte » (Jacques, 2007, p. 76), on pourrait dire, en parodiant le titre d'un roman de John Dickson Carr, que le lecteur est prévenu. L'abord d'un texte n'est jamais vierge de toute information et autre didascalie qui contribuent à en orienter la lecture - quand ce ne sont pas les lecteurs eux-mêmes qui font précéder leur lecture de cet avertissement qu'ils recherchent au titre d'un fil de trame. Ce qui n'est pas sans risquer de perturber leur compréhension; nous avons ainsi souvenir de l'élève qui, soucieux de connaître le contexte du passage à étudier, en avait oublié l'attention au texte lui-même: la compréhension du premier « valant » pour celle du second, dont il était incapable de citer une phrase ou un argument.

On le constate, la confusion guette, tant sur la façon de concevoir l'objet-texte que sur ce que suppose une compréhension du texte. Francis Jacques peut aider à la prévenir par une formule qui nous semble particulièrement heureuse : « une chose est de faire du texte un objet sémiotique avec ses polarités purement internes; une autre chose d'en faire un organon pourvu d'autonomie relative, non d'indépendance, par rapport au hors-texte, susceptible de s'interroger de manière immanente, mais aussi d'interroger la réalité » (Jacques, 2007, p. 15). Nous en retirons trois enseignements.

\subsection{Comprendre, c'est aussi « se » comprendre devant le texte}

9 Le premier est que pour mettre un apprenant en situation de compréhension d'un texte, on peut imaginer le lui présenter comme un "organon" à la fois au sens d'outil et d'ouvrage, c'est-à-dire matière qui se travaille, à la fois un moyen et un support: la présence du texte ne veut pas dire que tout est déjà donné, il reste des choses à faire advenir par un travail avec et sur lui. Ainsi il s'agirait, sinon d'éveiller, du moins de rendre attentif au fameux « moment de l'expression » dont parle Merleau-Ponty (1969) et dont nous pensons le recours éclairant, car il situe « la création du sens de l'œuvre » ni dans «l'opération d'écrire", ni dans «la lecture par autrui », mais dans le "moment fécond où le langage de l'auteur - ce langage parlant - m'interpelle et m'entraîne comme un tourbillon vers un nouveau sens que je vais rejoindre" (Merleau-Ponty, 1969, pp. 19-20).

10 Le deuxième enseignement concerne la pédagogie spécifique à développer sur la place de tout ce qui vient avec le texte sans être précisément le texte : la dynamique d'une œuvre - parfois d'un système philosophique - et bien sûr l'auteur lui-même. En vue de l'épreuve de philosophie du baccalauréat, les instructions officielles précisent que: " $\mathrm{La}$ connaissance de la doctrine de l'auteur n'est pas requise. Il faut et il suffit que l'explication rende compte, par la compréhension précise du texte, du problème dont il est question " (Bulletin officiel de l'Éducation nationale $n^{\circ} 31$ du 30 août 2012). Cette disposition nous semble à la fois très intéressante et très délicate à préparer. Car l'apprentissage des lettres comme celui de la philosophie se travaille le plus souvent en reliant un texte à son auteur, la présentation de ce dernier accompagnant, voire orientant la compréhension qui en fait un fil d'Ariane. Comment préparer à une rupture de méthode comme de repères pour le jour de l'épreuve, autrement dit comment désolidariser ce que l'enseignement, dans son souci de permettre la rencontre avec un auteur et une pensée, a toujours présenté selon une complémentarité nécessaire?

Le troisième enseignement nous conduit à penser la manière dont l'éducation au texte pourrait aider l'apprenant à vivre sa transformation, en tant que sujet-lecteur, dans l'expérience de la lecture: comment lui permettre d'aborder cette nouveauté de lui- 
même dans la confrontation à l'altérité du texte ? Ricœur pourrait ici encore s'avérer un précieux adjuvant.

Dans son ouvrage Du texte à l'action, Ricœur propose ainsi une certaine phénoménologie de l'acte de comprendre, comme deuxième temps d'une "triade situationcompréhension-interprétation » et qu'il fait apparaître comme une réflexivité fondée sur le texte: « Comprendre, c'est se comprendre devant le texte. Non point imposer au texte sa propre capacité finie de comprendre, mais s'exposer au texte et recevoir de lui un soi plus vaste, qui serait la proposition d'existence répondant de la manière la plus appropriée à la proposition de monde » (Ricœur, 1986, pp. 116-117). Comment le texte peut-il permettre une émergence du sujet-lecteur? Faut-il penser que l'un doit s'affirmer aux dépens de l'autre? Dans l'expérience de la lecture se jouerait un constructivisme singulier, puisque Ricœur nous laisse penser que ce qui caractérise un sujet-lecteur, ce n'est pas l'effort de (re)construire le texte pour lui-même, comme on peut reconstruire le discours d'autrui en substituant nos propres mots aux siens, pour saisir - c'est-à-dire traduire - ce qu'il veut nous dire. Il s'agirait plutôt de se laisser édifier par le texte, d'observer comment il nous restitue à nous-mêmes par le prisme de son altérité : «La compréhension est alors tout le contraire d'une constitution dont le sujet aurait la clé. Il serait à cet égard plus juste de dire que le soi est constitué par la "chose" du texte " (pp. 116-117). Dans ce que l'on pourrait qualifier de phénomène de renaissance continue par la lecture s'opère une « appropriation » du texte qui ne doit pas être confondue avec une annexion, autrement dit une réduction du texte à ce que je veux qu'il soit. La compréhension, indique Ricœur, " est alors autant désappropriation qu'appropriation » (pp.116-117) et elle requiert une suspension de soi («je ne me trouve qu'en me perdant»). Cette hypothèse de Ricœur relève d'une forme de décentrement " copernicien »: le texte n'est plus le seul donné soumis au lecteur, ce dernier se trouve exposé à lui-même par la médiation du texte, et à ce que la relation au texte va réorganiser de sa compréhension globale du monde. Dans une pédagogie du texte, la compréhension serait à promouvoir comme un processus double et solidaire, dont il ne faudrait pas hésiter à dire qu'il est troublant pour le lecteur, ne serait-ce que pour éviter à ce dernier d'associer ce trouble à un défaut qui proviendrait de sa manière d'être un lecteur. Il s'agit donc à la fois de "déployer la possibilité d'être indiquée par le texte " (Ricœur, 1986, p.127) et d'assumer, pour tenter de mesurer ce que ce déploiement occasionne de neuf chez le lecteur. C'est ici qu'une autre confusion, entre compréhension et interprétation, peut se profiler, écueil fréquent dans l'apprentissage du sujet-lecteur, et qu'il nous semble important d'expliciter pour espérer favoriser une édification du soi de l'apprenant à travers la lecture.

\section{La démarche interprétative au prisme de l'œuvre de Eco}

\subsection{Comment Umberto Eco pose le problème et les enjeux de l'interprétation}

Où se situe l'interprétation par rapport à la compréhension? "L'interprétation", propose Bordon, «qui dépasse la compréhension du sens dénoté, est le domaine de la construction d'un sens connoté, des inférences, de ce qui n'est pas forcément contenu dans l'explicite du texte mais est activé ou construit par le lecteur »(Bordon, 2004, p. 22). 
Ces éléments de définition esquissent aussi une problématique : quelle est cette matière que travaille l'interprétation et qui ne serait pas toute contenue dans le texte? Si l'interprétation fait entrer dans le "connoté ", comment y accède le lecteur? Peut-être parce que l'aider, c'est le mettre au pied du mur - ou du texte -, c'est ici, pensons-nous, qu'Eco peut se révéler un auteur et pédagogue original et efficace.

L'idée du nom de la rose me vint quasiment par hasard et elle me plut parce que la rose est une figure symbolique tellement chargée de significations qu'elle finit par n'en avoir plus aucune, ou presque : la rose mystique, et rose elle a vécu ce que vivent les roses, la guerre des Deux Roses, une rose est une rose, la vie en rose. Le lecteur était désorienté, il ne pouvait choisir une interprétation; et même s'il saisissait les possibles lectures nominalistes du vers final, quand justement il arrivait à lui, il avait déjà fait dieu quels autres choix. Un titre doit embrouiller les idées, non les embrigader (Eco, 1985, p. 9).

Lorsqu'il évoque ainsi l'idée du titre de son roman dans l'apostille, Eco se livre à une démarche singulière. Il indique sans détour ne pas souhaiter faciliter le travail interprétatif en usant d'un terme à la symbolique "saturée ", de manière à ne pas orienter l'interprétation tout en amenant le lecteur à ne pas renoncer à une réflexion sur le choix du mot. S'il y a une intention de l'auteur, c'est bien celle "d'embrouiller " pour ne pas «embrigader». Nous sommes bien dans un avertissement de l'auteur, qui ne cherche pas à ce que le lecteur ne se méprenne pas mais au contraire à ce qu'il se débatte avec une énigme, en ayant toutefois pris la précaution de lui confirmer que le titre en était bien une. Il y a bien une intention de l'auteur relative au choix du titre, mais elle n'est pas à appréhender comme « la » solution: il s'agit avant tout pour Eco d'exposer le lecteur à la non-univocité du sens et à l'expérience existentielle qui l'accompagne. La démarche se retrouve dans l'œuvre d'Eco - «Le soglie e l'infinito » (2007) notamment où il sensibilise à la perspective d'un sens sous-jacent au texte, caché un peu à la façon d'un deus absconditus sous sa forme intelligible. Il distingue en effet un sens littéral du texte, son intériorité propre, et les interprétations possibles, jamais désolidarisées d'un contexte, qui en font l'extériorité: habitudes interprétatives données par le contexte social, historique et culturel qui orientent le regard des sujets. Dès son Anti-dictionnaire philosophique, Chaudon, qui étudiait la démarche d'exégèse, en explicitait déjà les risques du côté du lecteur : « La confusion est toute entière dans le commentaire du philosophe et non dans le texte » (Chaudon, 1775, p. 408). C'est pourquoi nous pensons qu'Umberto Eco peut se révéler particulièrement précieux pour une éducation au texte et à la posture de lecteur : interpréter, c'est vivre, expérimenter, et trouver comment résoudre une tension entre l'intention de l'auteur, les possibles du texte et le lecteur qui tente de les rejoindre, en redoutant de leur faire violence et de s'imposer à eux, tout comme il redoute de les «manquer» tous les deux. En étayant la distinction entre «interprétation» et "surinterprétation ", il actualise également un problème posé par Meier et relevé par Greisch, à propos des lectures accommodatrices :

«Une accommodation (accommodatio) est un sens herméneutiquement faux qui ressemble très fortement au sens vrai [...] Nul interprète ne doit accommoder le texte, même si, par ailleurs, l'accommodation correspond à une séquence de représentations les plus parfaites. Une accommodation est un sens projeté sur le texte » (\$122). Le paragraphe 128 ajoute une précision intéressante : «Celui qui ne pense pas tout ce que l'auteur a pensé, ne le comprend pas correctement ; et celui qui pense plus que l'auteur, ne le comprend pas, parce qu'il postule un sens qui est partiellement faux ». L'interprétation peut donc échouer aussi bien en n'atteignant pas sa cible qu'en allant plus loin que la cible. Il y a ainsi deux manières, et non une seule, de ne pas rendre justice au texte : l'interprétation «surfaite », qui sollicite le 
sens par excès de bienveillance est aussi peu équitable que l'interprétation soupçonneuse, ou malveillante, qui ne fait pas confiance à l'auteur. Le juste milieu, que vise l'équité herméneutique, se situe entre un défaut et un excès (Meier, 1996, § 122 \& § 128, cité par Greisch, 2001, pp. 33-34).

Ce dont il convient de se garder, ce serait d'une double confusion: une conception de l'interprétation qui ne laisserait de place qu'au « soi » du lecteur et ferait de l'œuvre un prétexte pour dire ce que l'on voulait déjà dire sans elle, et d'autre part une conception qui ferait de l'interprétation la recherche d'un sens exclusif, lors même que le texte est particulièrement « ouvert " $(E c 0,1996)$ et qu'il encourage à ce qu'on pourrait appeler un «jeu» - au sens général d'interactions exploratoires - avec lui. Car si le texte n'oblige pas, il est ce qu'il est, c'est-à-dire l'instance qui permet sa propre interprétation; à ce titre, il est construit pour que le lecteur découvre comment s'y orienter. Eco précisera comment il conçoit l'exercice interprétatif : « Dans le De doctrina christiana, saint Augustin affirme qu'une interprétation paraissant plausible à un moment donné du texte ne sera acceptée que si elle est confirmée - ou du moins si elle n'est pas remise en question - par un autre point du texte. C'est cela que j'entends par intentio operis » (Eco, 1992, p. 39). Intégrer l'idée que le texte favorise une démarche interprétative qui se veut libre sans être hasardeuse conduit à envisager l'interprétation à travers une forme de coopération, déclinée en jeu et en négociation avec l'auteur.

\subsection{Vers une coopération textuelle : la métaphore de la partie d'échecs}

C'est une bien étrange relation qui s'inaugure avec l'entrée du lecteur dans un livre; le voici en présence du texte, relique d'une intention créatrice, et avec celle, non actuelle, de l'auteur, par l'intermédiaire de l'œuvre. Comment comprendre ce qui se joue dans une intersubjectivité différée et tâtonnante, actualisée par le texte comme tiers, entre le lecteur et l'auteur? Par nature "réticent", reconnaît Eco, le texte porte une virtualité décisive, qui appelle une coopération interprétative pour lui donner une "plus-value de sens» (Eco, 1979). Pour éviter l'« interprétation aberrante» (Eco, 1980) - expression proposée pour évoquer les torsions imposées à l'œuvre par des regards qui l'annexent en reniant l'intention spécifique et le contexte de son apparition - le texte doit laisser une ouverture qui rend possible un jeu, comparable à ce qui se produit dans une partie d'échecs, qui va permettre un positionnement réciproque, co-défini, du lecteur et de l'auteur. Eco explique en effet qu'une partie d'échecs est un ensemble de possibilités permises par la structure de l'encyclopédie du jeu d'échecs : le joueur/lecteur envisage toutes les possibilités objectivement reconnaissables comme admises et il choisit celle qu'il croit la meilleure, spéculant subjectivement sur la façon dont le joueur d'échecs réagira subjectivement aux possibilités offertes par le réseau. Comment pourra procéder le lecteur dans sa démarche de reconstitution d'une partie d'échecs entre Ivanov et Smith (Eco, 1979, pp. 175-177) telle que décrite par l'auteur de ce récit ? Pour comprendre ce qui a mené à cette situation ou issue de la partie, le lecteur pourra s'appuyer sur une connaissance objective, écrit encore Eco, qui touche aux possibilités admises par le jeu: ce préalable échappe encore à une intentionnalité, mais il est ce qui va l'organiser; et aussi, bien entendu, sur « ses propres spéculations subjectives quant au comportement d'autrui » (pp.175-177) tout de même appuyées par des signaux textuels, ruptures et disjonctions qui le placent dans une tentative d'anticipation de la suite. 


\subsection{La dialectique oscillation-négociation}

17 Ce qui constitue d'après Umberto Eco la "réticence " d'un texte est aussi son caractère fatalement inachevé, in-fini pourrait-on dire; le texte est une "machine paresseuse » dans la mesure où il suppose un travail coopératif du lecteur pour remplir les «blancs", tout ce que le texte laisse en suspens. Il y a pour le lecteur à faire l'apprentissage d'une "oscillation", propose Eco, laquelle traduit la recherche d'une mesure dans cet « équilibre instable entre initiative de l'interprète et fidélité à l'œuvre » (Eco, 1992, p. 18). Dans Lector in fabula, Eco préconise de ne pas aborder le texte seulement comme un ensemble de phrases. Si la compréhension travaille plutôt avec une "grammaire » du texte, ce dernier excède la somme de ses parties et c'est ce décalage qui rend possible l'interprétation. Ce faisant, l'interprétation pose la question de sa méthode et d'une mesure à lui donner, pour prévenir une « surinterprétation ${ }^{1}$ » qui renierait le texte en faisant l'espace de tous les possibles herméneutiques, par là de toutes les récupérations, ce qu'il ne peut ni ne saurait être. $\mathrm{Si}$ « d'aucuns se sont trop compromis sur le versant de l'initiative de l'interprète ", Eco estime que "le problème aujourd'hui n'est pas de se compromettre en sens inverse, mais bien de souligner une fois encore le caractère incontournable de l'oscillation" (Eco, 1992, p. 18). L'oscillation se conçoit ici de façon symbolique et comme processus, oscillation dans un rapport à soi et à l'auteur comme autre, oscillation entre un sens reconstitué du texte et les autres interprétations possibles dont il demeure le support. Cette oscillation est nécessaire - au sens philosophique d'inévitable - dans la mesure où «la rose dit toujours autre chose, et elle est toujours ailleurs. Elle est toujours plus et autre que la rose, mais, en quelque sorte, en silence » (Romanski, 2014, p. 107). Mais on ne peut pour autant s'en tenir à cette insaisissabilité, sous peine d'abdiquer le défi adressé au lecteur - et qui en caractérise la posture : «la rose requiert toujours une clef (c'est, selon le Rosarium philosophorum, au cœur même de la tradition alchimiste) ou tout au moins, une carte pour l'accompagner, la présenter, l'expliquer. C'est le jeu. C'est le jeu, la rose pose toujours problème» (p. 107). Eco va jusqu'à mettre en scène cette oscillation dans Le pendule de Foucault, parlant d'une « oscillation entre illusion fabulatoire et pressentiment d'un piège » $(\mathrm{Eco}, 1990, \mathrm{p} .50)$ et à en pratiquer lui-même une autre, dans Le Nom de la rose, par un "va-et-vient (...) entre une intrigue apparemment classique (conforme aux stéréotypes du roman d'énigme criminelle) et une narration subtile et caustique, truffée de références érudites et d'appels à la conscience intertextuelle » (Canvat \& Legros, 2004, p. 115).

L'oscillation chez Eco se conçoit comme une dynamique qui maintient un «aller vers » le texte et un retour réflexif consécutif à la rencontre du texte ; l'aller-retour doit permettre d'éviter une polarisation - qu'elle porte sur soi ou sur le texte - et ainsi de maintenir l'œuvre comme ce tiers vivant, qui rend possible une "coopération textuelle». Cette coopération suppose une certaine compétence "encyclopédique "-le texte entrant en résonnance avec d'autres œuvres - et se donne pour objectif de « retrouver je ne dis pas l'intention de l'auteur, mais l'intention du texte, ce que le texte dit ou suggère en rapport avec la langue dans laquelle il est exprimé et au contexte culturel où il est né » (Eco, 2007, p. 16). À travers ce qu'il envisage comme une oscillation, Eco permet au lecteur d'espérer échapper au piège de l'indécidabilité, qui le confinerait dans une inhibition face au texte, et de découvrir ainsi comment exister avec le texte selon une actorialité - faculté de se manifester comme acteur dans la vie d'une œuvre et de s'inscrire dans une triangulation auteur-œuvre-lecteur qui contribue à situer le propre de chacun. Prolongée dans 
l'exercice de la traduction, l'interprétation va donner lieu à une « négociation »; laquelle "étant justement un processus selon lequel, pour obtenir quelque chose, on renonce à quelque chose d'autre, et d'où, au final, les parties en jeu sortent avec un sentiment de satisfaction raisonnable et réciproque, à la lumière du principe d'or selon lequel on ne peut pas tout avoir» (p.16). À partir de ces séquences d'analyse, nous tenterons de proposer quelques éléments d'une approche formative du sujet-lecteur.

\section{Quelle approche pédagogique pour la formation du sujet-lecteur?}

\subsection{Consentir : éléments d'une éthique du lecteur}

19 Pourquoi envisager une "éthique » dans la formation d'un sujet-lecteur? La première raison est qu'il s'agit pour lui d'apprendre à se situer dans une triangulation constituée avec l'altérité du texte et l'autre d'un auteur, et que cet apprentissage ne s'inaugure que par un travail du sujet sur lui-même, nous dit Ricœur (1984). La deuxième raison tient à ce qui résulte de ce travail du lecteur sur lui-même, avec la prise d'une liberté qui lui est propre et qui doit trouver comment se manifester. C'est à partir de là, estime Ricœur, que l'éthique s'envisage comme une "odyssée de la liberté à travers le monde des œuvres, [un] voyage de la croyance aveugle ("je peux") à l'histoire réelle ("je fais") » (Ricœur, 1984, pp. 62-63). Il faut ensuite que cette liberté en quête de sa réalisation parvienne à adopter une posture dialogique avec l'œuvre et l'auteur, c'est-à-dire à s'affirmer sans les nier, pour parachever l'intention éthique dont parle Ricœur.

Un tel cheminement n'est pas si simple : aborder le texte n'est pas de facto en reconnaître l'altérité irréductible ; il est de nombreuses façons de s'en détourner, en le ramenant à du connu, en niant son mystère et la difficulté intellectuelle comme existentielle à laquelle il expose. L'entrée dans un texte s'inaugure par un premier consentement du lecteur : s'y exposer, pour apprendre de lui. "Même si le monde était un labyrinthe ", écrit Eco, «nous ne pourrions le traverser sans respecter certains parcours obligés » (Eco, 1992, p. 9) ; le premier de ces parcours est sans doute pavé d'humilité : consentir à rencontrer le texte pour le comprendre, consentir ensuite à cet « ensemble de possibilités permises par la structure d'une encyclopédie » (Eco, 1979) dans le cheminement interprétatif. Eco identifie lui-même un indispensable préalable: «il faut que le lecteur ait décidé de coopérer avec l'auteur ", coopération qui pour s'initier suppose d' " admettre que la partie Ivanov-Smith est à accepter comme la seule qui s'est effectivement produite » (Eco, 1979). Le premier moment-posture du lecteur s'inscrirait dans une éthique de l'acceptation de ce que le texte donne, tel qu'il semble le donner. Suivre un fil ou une méthode n'est pas contraire à l'expression d'une liberté ; le joueur d'échecs évolue sur un plateau limité, au moyen de pions dont le mode de déplacement est rigoureusement prédéfini, sans que cela ne compromette sa capacité à créer des combinaisons originales - voire géniales. Nietzsche le reconnaît lorsqu'il écrit sans détour, dans Par-delà bien et mal, que « la chose principale [...] c'est d'obéir longtemps, et dans une même direction. À la longue, il en résultait et il en résulte encore quelque chose pour quoi il vaut la peine de vivre sur la terre, par exemple la vertu, l'art, la musique, la danse, la raison, l'esprit - quelque chose qui transfigure, quelque chose de raffiné, de fou et de divin» (Nietzsche, 2000, p. 720). Apprendre suppose de se laisser conduire, le temps de se construire. C'est à cette condition que la nouveauté édifie, ou que l'on perçoit comment elle nous aide à s'édifier ; 
et si «les idées ne se séparent jamais de l'exemple qui les suggère », c'est à cette condition, sans doute, que peut apparaître le "modèle jamais entrevu jusqu'alors » (Levinas, 1968, p. 178) et que s'offre alors la richesse du texte.

\subsection{Savoir se perdre pour se trouver}

21 Pour devenir lecteur, peut-être faut-il d'abord s'être perdu face au texte. Que cela peut-il signifier? Dans la continuité de l'éthique que nous venons d'évoquer, rencontrer le texte suppose une disposition du lecteur à mettre en question ses repères, ce qui est souvent le cas une fois entré dans un labyrinthe. Mais le lecteur apprenant en a-t-il conscience ? La forme scolaire classique peut amener les apprenants à ne penser qu'à se situer dans une adéquation/restitution : que faut-il en dire d'attendu, ce qui peut devenir un «que faut-il en re-dire»: posture qui favorise la paraphrase, peut compromettre un approfondissement de la relation à l'œuvre. Se perdre, c'est aussi assumer l'obscurité des commencements; à Dupin qui se voit soumettre une énigme dans La Lettre volée, Edgar Poe fait dire que "nous l'examinerons mieux dans les ténèbres » de sorte qu'il refuse d'éclairer la pièce. Se perdre, cela peut signifier que l'on accepte l'énigme et que l'on se rend disponible à ce qui advient par la lecture du texte. Une disponibilité authentique du lecteur facilitera une prise de conscience des différentes oscillations qui jalonnent son cheminement tâtonnant vers le sens; "par exemple, il induit les propriétés psychologiques d'un personnage à partir de ses actes, puis anticipe des comportements futurs à partir de ces propriétés » (Bayart, 2007, p. 27). Se perdre, c'est donc peut-être travailler la matière du texte de façon tâtonnante au risque des "promenades inférentielles » (Eco, 1979, p. 151), « ces échappées hors du texte, pour y revenir riche d'un butin intertextuel » (p. 151).

\subsection{Découvrir et s'approprier une autorialité singulière}

Qu'il s'agisse de se faire lecteur ou bien de mener un travail d'écriture, un certain nombre d'élèves comme d'étudiants éprouvent souvent des difficultés à concevoir ce que représente "être auteur». La rencontre avec des auteurs est souvent une étape importante dans l'apprentissage de son positionnement de «sujet» face au texte. L'auteur y est présent selon un clair-obscur; il est là à travers son texte, mais son intention ne se laisse pas saisir aisément. «Tu essayais de les tirer à toi. Mais ils ne se laissent guère tirer» (Deleuze \& Parnet, 1977, p. 32) ; parfois considérés comme les démiurges du texte, uniques détenteurs de la «solution» et seules normes de l'interprétation, les auteurs n'inspirent guère spontanément la perspective d'une coopération dont le texte serait le médium. Or, se découvrir comme lecteur c'est aussi, nous semble-t-il, s'éveiller à une part d'autorialité, c'est-à-dire à la responsabilité de pouvoir faire quelque chose du texte qui le prolonge comme œuvre à travers l'espace que ménage ce texte et auquel il faut sensibiliser le lecteur en quête de lui-même. Pour éclairer cette part d'autorialité, revenons à la métaphore du jeu d'échecs proposée par Eco. La démarche du joueur-lecteur, fondée à la fois sur une généalogie de la narration et de l'intention de l'auteur, va se retrouver face à un dualisme de possibles: «De deux choses l'une », écrit Eco : « soit il a deviné, soit il n'a pas deviné ». Notons qu'Eco n'écrit pas «il a échoué » à deviner, ce qui souligne que cette recherche d'une forme d'adéquation n'existe que dans le temps de cette relation consentie avec le travail de l'auteur, et tant que dure ce consentement : on n'y est obligé que dans la mesure où l'on 
consent à s'y essayer. Confronté à l'inadéquation constatée (ce n'est pas la trame retenue par l'auteur), le lecteur, poursuit Eco, pourra jeter « (avec dépit), sa fiche parce qu'elle constitue la représentation d'un possible état de choses que le cours de la partie (considérée comme la seule bonne) n'a pas confirmé» (Eco, 1979, p. 151). Le paradoxe, c'est que l'inadéquation, finalement avérée, de son hypothèse une fois confrontée à la "solution» de l'auteur n'invalide pas pour autant son caractère plausible: «l'état alternatif qu'il avait prévu peut être parfaitement admis du point de vue des échecs; il était tout à fait possible et il l'était si bien que le lecteur l'a effectivement représenté ». C'est là ce qui en fait une expérience potentiellement stimulante qui mènerait le lecteur ailleurs, à basculer dans une autorialité clairement assumée : «on peut interrompre un roman policier au beau milieu pour écrire son propre roman, sans se préoccuper de savoir si le cours des événements que l'on a imaginé coïncide avec celui que valide l'auteur » (Eco, 1979, p. 151). Suivre une pensée qui se développe au fil d'un livre présente une possibilité d'émancipation pour le lecteur, qui peut advenir par un texte dont il choisit de faire l'heuristique de son propre projet, aperçu grâce à la médiation de l'œuvre autre.

Dire que le texte est ce qu'il est, une fois donné à lire, ce n'est pas affirmer qu'il a atteint les limites de son être et que ce dernier ne continue plus à se déployer. Bien au contraire : il entre dans une phase de son existence où il continue à s'édifier en édifiant. C'est, littéralement, une existence qui «fait » débat: en se donnant tel qu'il est écrit, le texte nourrit un discours sur le sens qu'il initie, comme il entretient par ailleurs une réflexion sur ce qu'il donne à penser. Il ne prétend donc pas à l'achèvement - quoiqu'avec sa publication, sa forme ait acquis un caractère définitif - et ce faisant, il fait signe au lecteur. C'est sur ce « faire signe » qu'une confusion peut déjà s'installer. Le texte n'initie pas de " concurrence existentielle », où il s'agirait pour le lecteur comme pour le texte de " résister » en s'affirmant aux dépens d'une altérité. Au contraire, c'est le "faire signe » d'une possibilité d'édification par jeu de regards. Lorsqu'il écrit que «la cohérence du texte n'est pas une chose qu'il possède avant d'avoir été décrit » (Rorty dans Eco éd., 1996, p. 89), Rorty laisse comprendre que le texte espère un achèvement par sa lecture. Mais à ce jeu, il participe à l'édification de son lecteur, auprès de qui il renouvelle l'énigme du sens ; sans doute est-ce pour cela qu'Eco écrit qu' «il n'est rien de plus significatif qu'un texte qui affirme son divorce d'avec le sens» (Eco, 1992, p.18). Nous avons tenté de proposer quelques pistes qui peuvent guider l'apprentissage d'un sujet-lecteur à la découverte de son rôle, découverte qui commence sans doute par dissiper une confusion fondamentale sur ce que lire "veut dire", sur l'incertitude nécessaire qui va avec une rencontre du texte et sur cette édification réciproque qu'elle rend possible. Car la lecture, pour reprendre une formule de Deleuze, «c'est aussi bien l'envers : comment la pensée peut secouer son modèle, faire pousser son herbe, même localement, même dans les marges, imperceptiblement [...] des pensées qui ne s'exerceraient pas dans une concorde des facultés, mais qui porteraient chaque faculté à la limite de sa discordance avec les autres, qui ne se fermeraient pas sur la recognition, mais s'ouvriraient à des rencontres, et se définiraient toujours en fonction d'un Dehors, qui n'auraient pas à lutter contre l'erreur, mais auraient à se dégager d'un ennemi plus intérieur et plus puissant, la bêtise »(Deleuze \& Parnet, 1977, p. 33). 


\section{BIBLIOGRAPHIE}

Baudelaire, C. (1993). Euvres complètes (II). Paris : Gallimard.

Bayart, D. (2007). Sur les aspects logiques de l'interprétation des signes chez Peirce et Eco, Le

Libellio d'Aegis, 3/4, 24-34.

Bordon, E. (2004). L'interprétation des pictogrammes. Approche interactionnelle d'une sémiotique

(pp. 21-22). Paris : L'Harmattan.

Chaudon, L-M. (1775). Anti-dictionnaire philosophique. Paris : Saillant et Nyon.

Danvers, F. (1994). 700 mots-clés pour l'éducation. Lille : Presses universitaires de Lille.

Dufays, J-L. (2004). « La dialectique des valeurs : le jeu très ordinaire de l'évaluation littéraire ", dans K. Canvat et G. Legros, Les valeurs dans/de la littérature (pp. 103-121). Namur : Presses universitaires de Namur.

Deleuze, G. \& Parnet, C. (1977). Dialogues. Paris : Flammarion.

Eco, U. (1979). Lector in fabula. Le rôle du lecteur ou la coopération interprétative dans les textes narratifs

. Paris : Grasset.

Eco, U. (1980). Towards a Semiotic Enquiry into the Television Message, dans J. Corner \& J. Hawthorn (éds.), Communication Studies: An Introductory Reader (pp.131-150). Londres : Edward Arnold.

Eco, U. (1985). Le Nom de la rose. Paris : Grasset.

Eco, U. (1990). Le pendule de Foucault. Paris : Grasset.

Eco, U. (1992). Les limites de l'interprétation. Paris : Grasset.

Eco, U., Rorty, R. \& Culler, J. (1996). Interprétation et surinterprétation. Paris : PUF.

Eco, U. (2007). Le soglie e l'infinito, dans C. Paolucci (dir.), Studi di semiotica interpretativa

(pp.145-176). Milan : Bompiani.

Eco, U. (2016). Écrits sur la pensée au Moyen Âge. Paris : Grasset.

Greisch, J. (1987). Le texte comme objet philosophique. Paris : Beauchesne.

Greisch, J. (2001). Le principe d'équité comme « âme de l'herméneutique » (Georg Friedrich Meier), Revue de métaphysique et de morale, 1(29), 19-42.

Greisch, J. (2006). Entendre d'une autre oreille : les enjeux philosophiques de l'herméneutique biblique. Paris : Bayard

Jacques, F. (2007). L'arbre du texte et ses possibles. Paris : Vrin.

Kierkegaard, S. (1963). Journal. Paris : Gallimard.

Levinas, E. (1968). Quatre leçons talmudiques. Paris : Minuit.

Meier, G.F. (1996). Versuch einer allgemeinen Auslegungskunst. Hambourg : Meiner.

Merleau-Ponty, M. (1969). La prose du monde. Paris : Gallimard.

Nietzsche, F. (2000). Par-delà bien et mal. Paris : Flammarion. 
Raxhon, P., Rainkin, M. \& Godeaux, B. (2006). Une introduction à la neutralité active. Liège : Céfal.

Renard, J-C. (1970). Notes sur la poésie. Paris : Seuil.

Ricœur, P. (1984). Fondements de l'éthique, Autres temps, 3, 61-71.

Ricœur, P. (1986). Du texte à l'action. Paris : Seuil.

Romanski, P. (2014). Poétique d'une reconstruction. Lille : Presses universitaires de Septentrion.

Rouzel, J. (2016). Posture d'éduc..., dans B. Bouchereau, La posture éducative (pp. 9-19). Toulouse :

Érès.

Vandendorpe, C. (1991). Lecture et quête de sens, Protée, 19(1), 95-101.

Vandendorpe, C. (1999). Du papyrus à l'hypertexte : essai sur les mutations du texte et de la lecture.

Paris : La Découverte.

Wolff, C. (1736). Logique, ou réflexion sur les forces de l'entendement humain et sur leur légitime usage dans la connaissance de la vérité. Berlin : A. Haude.

\section{NOTES}

1. On peut remarquer à ce propos qu'une surinterprétation, conçue comme surenchère dans un discours sur l'au-delà du texte, pourrait fort bien conduire à se perdre de vue elle-même et à revenir ainsi au texte. Avec finesse, Dufays note en effet que « une fois atteint un stade de saturation dans la recherche de la rupture et de l'originalité, une ultime rupture encore possible consiste à rompre avec la rupture, non pour adopter la posture inverse et retomber dans l'attitude classique, mais pour adopter une attitude dialectique, fondée sur l'oscillation, le va-etvient » (Dufays, 2004, p. 115).

\section{RÉSUMÉS}

Lire s'apprend; mais apprend-on à devenir lecteur? Être un lecteur suppose une manière d'aborder le texte, de concilier un art de la présence et de la juste distance face à l'altérité du texte et à l'autre de l'auteur. Inspiré par des observations menées auprès de lycéens, cet article propose de réfléchir sur la manière de former à une posture de lecteur. Une telle posture nécessite de dissiper la confusion qui entoure souvent compréhension et interprétation du texte et elle conduit le lecteur à se comprendre lui-même au prisme du texte. Elle se construit à partir d'une démarche éthique ricœurienne qui doit trouver comment concilier l'expression de la subjectivité du lecteur, l'intégrité du texte et la liberté créative de l'auteur ; comme le suggèrent les travaux d'Eco, elle confère au lecteur une forme d'autorialité par laquelle il s'édifie en prolongeant l'œuvre.

INDEX

Mots-clés : lecteur, posture, interprétation, compréhension, autorialité 


\section{AUTEUR}

\section{EMMANUEL NAL}

Maître de conférences en sciences de l'éducation à l'université de Haute-Alsace, chercheur au LISEC (EA 2310) 